\title{
Paleopathology of the commoners at Tell Amarna, Egypt, Akhenaten's capital city
}

\author{
Jerome C Rose
}

Anthropology Department, University of Arkansas, Fayetteville, Arkansas, 72701, US

The Egyptian Pharaoh Akhenaten initiated worship of a single god and established a new capital city (Tell Amarna) that was built and occupied only once from 1350-1330 BCE. This single short occupation offers a unique opportunity to study a short time period. The royal tombs have long been known and studied, but the location of graves for the common inhabitants has been an archaeological puzzle for more than 50 years. Recently four cemeteries have been located and the analysis of commingled bones from the South Tombs cemetery is presented here. The remains yield the following demographic profile: 53 adults with 19 females and 18 males; 14 juveniles between the ages of 5 and 17; and 3 infants. Arthritis and degenerative joint disease of the spine and joints indicates that DJD was not excessive. Only 2 to $8 \%$ of the adult population exhibits arthritis. There are 3 healed fractures of the arm (2 to $8 \%$ of the adult sample). There is 1 healed compressed fracture of the skull suggesting violence. The adult infection rate is between 2 and $8 \%$ with 3 healed and 1 active case of periostitis and no severe infections. Anemia is implicated by $23 \%$ of adult frontals exhibiting cribra orbitalia. Life for the common residents of Amarna appears to not have been as good as initially postulated.

Key words: paleopathology - ancient diseases - diseases in antiquity - human remains

Akhenaten, Pharaoh of Egypt, instituted worship of a single god to replace the traditional complex pantheon. In 1350 BCE he founded a new capital, now known as Tell Amarna, located between the previous capitals of Memphis (near Cairo) in the north and Thebes (modern Luxor) in the south (Kemp 1991). This city was created on previously unused land along the Nile and was occupied for approximately 20 years, when it was then abandoned upon Akhenaten's death. Amarna is unique among cities of the ancient world because it did not develop over a long period of time with one building cutting into the one previous, but was planned and built upon vacant land and occupied by approximately 30,000 people for less than 20 years.

The tombs of the nobles were either left unfinished or were emptied and the contents moved to the traditional burial locations across the river from Thebes. Although excavated since 1892 no cemeteries of the middle class or workers have been found. Repeated searches have failed to find the bones of the estimated 6000 dead (Griffith 1923) until recently. A cemetery located along the wadi adjacent to the South Tombs (high status) was washed out in a past flood leaving the bones and pottery scattered along the surface of the wadi. This event offered the opportunity to examine the skeletal remains of the Amarna lower class inhabitants. The bones were collected along the wadi and its alluvial fan and brought to the on-site laboratory

Research conducted as part of the Armara Project of the Egypt Exploration Society directed by Barry Kemp University, Cambridge

E-mail:jcrose@uark.edu

Received 20 July 2006

Accepted 16 October 2006 where they were sorted into groups of diagnostic bones and examined for indicators of minimum number of individuals, age and sex, and evidence of disease.

The paleopathology of Amarna is interesting because of the unique circumstances of the city and three hypotheses are tested. First, having been built on new land and occupied for only a short period of time, contamination of the environment with human waste and parasites should have been minimal and, thus, bacterial infections and parasites should have been infrequent. Second, as a capital city it is expected that food sources should have been at least adequate for all inhabitants. Third, as a city under construction skeletal lesions related to heavy work loads and accidents should have been high.

\section{MATERIALS AND METHODS}

Nine numbered tile markers were distributed south (1) to north (9) down the wadi adjacent to the South Tombs area. Tile 1 was situated adjacent to the southern portion of the cemetery located on the east wadi bank and tile 9 was located at the mouth of the wadi. The wadi alluvial fan was designated as the area "west of marker 9". All human bones and ceramic sherds were collected by a walking survey. The collections were marked by location such as between markers 1 and 2 .

The human bones were cleaned by dry brushing and examined. No differences were observed between bones collected between any of the markers and, thus, for interpretation the data were combined. The bones collected from the wadi fan (west of 9) and the bones from the wadi proper were analyzed and are presented separately. No differences were found and the data are combined for the final interpretation.

Each bone or bone fragment was examined for diagnostic characteristics of age and sex, as well as pathological lesions. The following diagnostic bones or skeletal elements were separated out for a second detailed analy- 
sis: brow ridges and superior eye orbits; temporal bones having a minimum of the external auditory meatus; occipital with a minimum of the protuberance; any parietal fragment that might exhibit porotic hyperostosis; all maxillary and mandibular fragments and all teeth; clavicle fragments greater than $50 \%$; head and distal ends of humeri; head and distal ends of radii; some of the complete bones of the hands and feet; the odontoid process of the second cervical vertebrae; fragments of ileum if the sciatic notch and or auricular surface is present; the proximal end of the femur if the neck and lesser trochanter are preserved or the distal end if complete; the proximal and or distal ends of the tibiae if complete; the proximal and distal ends of the fibulae if complete; any bone fragment that exhibits a pathological lesion. All bones meeting these criteria were subjected to detailed examination one bone category (i.e., humerus heads) at the same time with all the fragments arranged on the work table. Most of the teeth had fallen out of the mandibular and maxillary fragments and only a few teeth were found while collecting in the wadi. The following information was collected by examination of the tooth sockets in the jaw fragments: tooth present at death, tooth lost before death, tooth abscessed due to decay or wear.

\section{RESULTS}

\section{Minimum numbers}

Frontal bone of the skull - In 1-9 there are 23 frontal bone fragments with sufficient portions of the nasal and brow ridge component preserved that they could be ranked by size and robustness. Using this seriation 10 were judged to be females and 13 males for the minimum adult sample of 23. In west of 9 there are 9 frontal fragments with 4 being female and 5 male. Combined there are 32 adult frontals representing 14 females and 18 males.

Occipital bone of the skull - In 1-9 there are only 8 relatively complete occipital protuberances and all of these scored male. In west of 9 there are 9 scorable protuberances and 5 are female and 4 male. Combined there are 5 females and 12 males.

Temporal bone - The petrous portion of the temporal bone is solid and survives well, often preserving the external auditory meatus. In 1-9 there are 26 left and 25 right adult unique temporal portions. This provides a minimum number of 26. There are 17 right mastoids that could be scored to indicate 11 females and 6 males. In west of 9 there are 29 left and 28 right unique temporal bones for a minimum number of 29. Of the left mastoids 7 are scored female and 4 male. Combined there are 55 adults of which 18 are females, 10 are males, and 27 of unknown sex.

Mandible and teeth - The combined sample of mandibles provides a minimum number of 40 adults (13 females, 10 males, and 17 unknown) and 5 children with the following ages at death: $1.5,5,8,8.5$, and 10 years. There were a total of 272 observable tooth sockets and $18 \%$ of these showed evidence of premortem tooth loss (49/272), while $3.7 \%$ showed evidence of abscess (10/272).

Maxilla and teeth - The two collections combined produced 265 observable maxillary tooth sockets and $6.8 \%$ of these showed evidence of premortem tooth loss (18/ $265)$, while $1.9 \%$ showed evidence of abscess (5/265). There are fewer maxillary teeth missing premortem than mandibular.

Mandible, maxilla, and teeth combined - Combining mandibular and maxillary teeth there are 36 adult maxillary dental sets and 6 juveniles with the following ages at death: $5,9,11,15,17$, and 17 years. Using both the maxillary and mandibular teeth there is a combined minimum number of 40 adults and nine aged subadults with the following ages: $1.5,5,8,9,10,11,15,17$, and 17 years. Combining the maxillary and mandibular tooth socket data produces a premortem loss rate of $12.5 \%(67 / 537)$ and an abscess rate of $2.8 \%(15 / 537)$.

Axis vertebra - In 1-9 there are 7 adult axis vertebrae for a minimum number of 7 adults. In west of 9 there are 5 axis vertebrae for a minimum number of 5 . The combined minimum number is 12 .

Clavicle - In 1-9 there are 8 left and 10 right adult and 3 right juvenile clavicles. The minimum number is 10 adults and 3 juveniles. In west of 9 there are 13 adult and 2 infant left clavicles, while there are 12 adult, 1 juvenile, and 1 infant right clavicles. The minimum number is 13 adults, 1 juvenile, and 2 infants. The combined minimum number is 23 adults, 4 juveniles, and 2 infants. The sizes range from large to small, but there is no very large male example.

Humerus -In 1-9 there are 24 unique superior portions of the olecranon fossa of the left humerus (distal ends) with no immature individuals. There are 8 adult right distal humeri and 4 juveniles. The minimum number is 24 adults and 4 juveniles. One complete humerus provides a height estimate of $152 \mathrm{~cm}$ if a female or $157 \mathrm{~cm}$ if a male. West of 9 has 17 left and 14 right superior portions of the olecranon fossa of the humerus. The minimum number is 17 adults. The combined minimum number is 41 adults and 4 juveniles. The range in size of the various portions of the humerus indicate that there are both males and females, but that the largest males are smaller than other males from various components of the Amarna skeletal sample.

Ulna - In 1-9 there were 11 adult right ulnae, 2 juvenile right ulnae, 19 adult left ulnae, 2 juvenile left ulnae, and 1 infant left ulna. There is a minimum number of 22: 19 adults, 2 juveniles, and 1 infant. West of 9 there are 22 adult right ulnae, 1 juvenile right ulna, and 1 infant right ulna, and 23 adult left ulna. The minimum number is 23 adults, 1 juvenile, and 1 infant. Combined there are a minimum number of 42 adults, 3 juveniles, and 2 infants. The range in size of the ulna heads suggests that there are both males and females in both collections and that the sexual dimorphism is the same in both. Male ulnae are not extremely large in size (as seen elsewhere among the Amarna collections), nor are the females extremely small.

Radius - In 1-9 there are 6 left, 7 right and 5 unsidable adult proximal radii for a minimum number of 7 . West of 9 there are 10 left, 13 right, and 3 unsidable adult radii for a minimum number of 13 . Combined there are 20 adults represented. The range in size is similar to the ulnae, but at 
least one large rugged radius head was found.

Ilium - In 1-9 there are 8 adult ( 4 females, 3 males, and 1 unknown) and 2 immature portions of the right ilium. There are 2 adult (both females) and 1 immature left ilium fragments. The minium number is 8 adults (at least 4 females and 3 males) and 2 juveniles. West of 9 there are 5 adult ( 4 females and 1 male), 3 juvenile, and 1 infant left iliae. The are 2 right adult iliae (both males). The minimum number is 6 adults ( 4 females and 2 males), 3 juveniles and 1 infant. The combined total is 14 adults ( 8 females and 5 males), 5 juveniles, and 1 infant.

Femur - In 1-9 there are 5 adult and 5 juvenile left femora and 10 adult and 2 juvenile (one matched pair is counted in both) right femora. The minimum number is 10 adults and 5 juveniles. The complexity of the femora enables reliable right and left matching and thus it is possible here to determine a more complete minimum number to be 15 adults and 6 juveniles. In west of 9 there are 8 adult and 1 infant left femora and 4 adult and 6 juvenile right femora for a minimum number of 8 adults, 6 juveniles, and 1 infant. Matching provides for a refined minimum number 13 adults, 6 juveniles, and 1 infant. The combined minimum is 28 adults, 12 juveniles and 1 infant.

Tibia - Unique portions of the tibiae are poorly represented. There are 4 proximal tibia ends and at least 2 nonmatching left distal adult ends. There is 1 adult and 1 juvenile right tibiae. There is a minimum of 6 adults, and 1 juvenile. In west of 9 there are 8 left and 4 right adult tibiae. The minimum number is 8 adults, but again morphology is sufficiently complex for right and left matching that demonstrates a refined minimum of 12 adult individuals. Combined the minimum number is 18 adults and 1 juvenile. There are three tibia that can be measured for height calculation. The smaller person would have been $149 \mathrm{~cm}$ if a female or $154 \mathrm{~cm}$ if a male. The next taller would have been $160 \mathrm{~cm}$ if a female or $163 \mathrm{~cm}$ if a male. The tallest would have been $167 \mathrm{~cm}$ if a female or $170 \mathrm{~cm}$ if a male.

Fibula - In 1-9 there are 4 right and 2 left adult fibulae for a minimum of 4 adults. In west of 9 there are 2 left distal ends for a minimum of 2 . The combined minimum number is 6 adults.

\section{Comprehensive minimum numbers}

In the 1-9 collection the greatest number of unique adult bones is the temporal with 26 . The largest number of female bones is 11 temporals, while the largest number of male bones is 13 frontals. This suggests that there are at least 11 females and 13 males in this collection. There are 6 unique juvenile femora. The maxillary teeth provide ages at death for 4 of these 6 juveniles of 5, 9, 11, and 15 years. Two juveniles' ages at death must remain unknown. There is one infant represented by an ulna.

In the west of 9 collection the greatest number of unique adult bones is also the temporal with 29. As we have 2 juveniles of 17 years each and it is not possible to differentiate their temporal bones from the other adults, the total number of adults is reduced by 2 and the minimum number of adult individuals is 27 . There are a minimum number of 8 females and 5 adult males. Again we have 6 juvenile femora, however the two 17 year olds would have had fused femoral heads and would not have been classified as juveniles, thus the number of juveniles must be increased by 2 producing a minimum number 8 . Four of the eight juvenile ages are known from the teeth: $8,10,17$, and 17 . There are 2 infant clavicles for a minimum number of 2 infants. One infant age is known to be 1.5 years from the teeth.

Combining both bone collections provides that there is a minimum number of 53 adults with at least 19 females and 18 males. The pubic symphyses indicated that one male and one female were 35-39 years old at the time of death and one adult of unknown sex was 30-35 years old. There are at least 14 juveniles with the ages of eight of them known: $5,8,9,10,11,15,17$, and 17 years. There is a minimum of 3 infants with one known to be 1.5 years from dental development. The demographic information indicates no difference between these two artificial collections (separated by a walkway built for tourists) and, thus, both collections should be interpreted as a single unit.

\section{Paleopathology}

All pathological and abnormal features observed in the skeletal collection are described in this section. The minimum number analysis above showed that there is no meaningful differences between the 1-9 and west of 9 skeletal collections and, thus, they are considered together for the interpretation of the paleopathology. As each category of skeletal abnormality is discussed the percentages of potentially effected people are presented by both the number of bones observed and the minimum number of 53 adults.

All joint surfaces were examined for evidence of arthritis and other degenerative disease. No abnormalities were seen on the joint surfaces of 42 ulnae, 20 radii, 41 humeri, 14 acetabula (hip), 28 femora, and 18 tibiae. One glenoid surface of a right scapula shows destruction of the joint surface exposing the underlying trabecular bone. This one case suggests a shoulder joint arthritis rate of $2 \%(1 / 53)$. One right patella shows extensive pitting on the superior medial joint surface. There are only 13 patellae available for examination suggesting a $8 \%$ degenerative disease rate or $2 \%$ if the minimum number of 53 is used. Both these lesions show extensive pitting and destruction of the joint surface indicating prolonged and hard use of these joints. Terminal phalanges of two big toes, and two middle toes show extensive lipping of the joint margins. This might have been caused by long term kneeling.

The vertebrae of the spine also show only a few signs of deterioration. Two cervical (neck vertebra) have three dimensional osteophytes along the margins of the body which also show compression of the body. One thoracic and two lumbar vertebrae have small osteophytes along the body margins. Four lumbar vertebrae have three dimensional deposits of osteophytes that are sufficiently extensive to have a hook appearance. Two thoracic and one lumbar vertebrae have Schmorl's nodes indicating herniated discs. The amount of back stress would range between 2 and $8 \%$ of the adult population.

There are three healed broken arm bones. One ulna of 
42 examined has a well healed fracture indicating a $2 \%$ fracture rate. One radius of the 20 examined has healed fracture of the proximal end that is only slightly misaligned after healing. The 41 humeri examined, one distal end of a right humerus shows misalignment after healing. This misalignment would have interfered with normal functioning of the elbow. The fracture rate would vary between 2 and $6 \%$. All of these fractures are most likely due to an accident involving the elbow. There is one compressed circular fracture $5.5 \mathrm{~mm}$ in diameter found on an occipital bone that might have come from interpersonal violence. This circular fracture and the crack in the bone radiating out from it are healed indicating that the blow causing the fracture occurred long before death. Based on 53 adults the trauma from conflict rate is $2 \%$.

The most common bone infections are caused by bacteria found throughout the environment. One left tibia shows the new bone formation indicating active infection (periostitis) at the time of death. Another left tibia shows evidence of healed periostitis as does a fragment of a tibia and a long bone fragment that might be a humerus. These lesions show bone that is being remodeled indicating that the infection had been countered some months to years before death. The active infection rate is $6 \%$ based on 18 observed tibia or $2 \%$ based on a total of 53 adults. Counting all 4 infections including active and healed produces an infection rate of $8 \%$ based on 53 adults.

There are two small lytic lesions where the bone had been removed by the inflammatory process leaving a smooth cavity. One is found in the proximal joint surface of the second phalange of the big toe and the other on odontoid process of the second cervical vertebra.

Seven of the 30 adult orbits examined had cribra orbitalia producing an anemia rate of $23 \%$. Three of the 90 large parietal fragments had extensive large pits and thickening indicative of porotic hyperostosis. Using the minimum number of adults provides a rate of $6 \%$. Minor pitting was common and observed on $23 \%$ of the fragments $(20 / 90)$, but these have not been scored here as porotic hyperostosis. The anemia rate should range between 6 and $23 \%$.

There was only one benign cancer found. An osteoma $6.4 \mathrm{~mm}$ in diameter and $2.4 \mathrm{~mm}$ in height is located just to the left of the chin of an adult.

\section{Conclusions}

The analysis of the South Tombs skeletal material washed out into the wadi yields the following demographic profile: 53 adults with 19 females and 18 males; 14 juveniles between the ages of 5 and 17; and 3 infants. Infants are clearly under represented. It is possible that their small bones did not survive to be found or that they were never in the cemetery. On the other hand $20 \%$ juveniles is too high. The ages between 5 and 17 years should have the very lowest mortality. This demographic distribution suggests that life for the commoners at Amarna was hard and stressful.

Rates of arthritis and degenerative joint disease of the spine and major joints are not high with only 2 to $8 \%$ of the adult population exhibiting these lesions. The lesions of the scapula and patella are more serious and involve destruction of the joint surface. Considering the few vertebrae that could be examined the incidence of Schmorl's nodes could be significant. Broken bones that have healed are also only 2 to $8 \%$ of the adult sample. These trauma of the elbow region are most likely due to work accidents. Taken together the degenerative joint lesions, Schmorl's nodes, and healed fractures all suggest that work loads are high. This is not unreasonable considering that this was a city under construction.

The adult infection rate is between 2 and $8 \%$ which is relatively low with no severe infections being observed. This is within the range expected. However, $23 \%$ of the 30 adult eye orbits exhibit cribra orbitalia which exceeds the hypothesized level. In comparison, we find that New Kingdom to Late Period Nobles from Thebes had a mean cribra orbitalia rate of $29.2 \%$ (Zink et al. 2000), while adult rates from early and late Christian cemeteries at Kulubnarti, Nubia, ranged between 18 and 50\% (Van Gerven et al. 1995), and Late Period and Early Christian adults from Dakhleh Oasis, Egypt, vary between 39 and 84\% (Fairgrieve \& Molto 2000). Although on the low end of this range, the Amarna cribra orbitalia frequency is not unusual for Egypt. The high rates for Cribra Orbitalia in Egypt and Nubia have been attributed to a complex synergism of disease, parasitism and a diet deficient in bio-available iron (e.g., Van Gerven et al. 1995), but the postulated cleaner environment of Amarna suggests that the causes were more complex. Fairgrieve and Molto (2000) add folic acid deficiency to the equation. Since folic acid is destroyed in the capillaries of the skin by ultraviolet light, and since living in Egypt means living in plentiful sunlight with little moisture to filter out the ultra violet, then the folic acid content of the ancient Egyptian diet needs a careful examination.

In conclusion, the South Tomb bones from Amarna suggest that life was sufficiently stressful to increase juvenile mortality; the work loads high enough to result in spinal and limb trauma; and the diet not sufficiently adequate to resolve the problem of anemia.

\section{REFERENCES}

Fairgrieve SI, Molto JE 2000. Cribra orbitalia in two temporally disjunct population samples from the Dakhleh Oasis, Egypt. Amer J Phys Anthropol 111: 319-331.

Griffith FL 1924. Report on the 1923 season at Tell Amarna. $J$ Egyp Archaeol 10: 303-304.

Kemp BJ 1991. Ancient Egypt: Anatomy of a Civilization, Routledge, London.

Van Gerven DP, Sheridan SG, Adams WY 1995. The health and nutrition of a Medieval Nubian population. Am Antropol 97: 468-480.

Zink NA, Hagedorn HG, Szeimies U, Weyss C 2000. Anthropological and palaeopathological analysis of the human remains from three "Tombs of the Nobles" of the Necropolis of Thebes-west, Upper Egypt. Anthropol Anzeiger 58: 321343. 